enabling arrayed biomolecules (DNA or proteins) to keep their native conformation. Probe biomolecules are mixed with the prepolymer, and nanolitre droplets are arrayed on a glass slide. During curing, covalent reactions simultaneously crosslink the prepolymer, tether the biomolecules to the gel matrix and bind the droplets to the slide. The result is many levels of probes, giving high sensitivity and a broad dynamic range. Biocept has developed genomic arrays and protein arrays are coming soon.

\section{Mapping interactions}

"If you are talking proteomics, what you are looking for must not be predefined, it has to be an open approach," says van Oostrum. Reverse arrays, where the cell extract itself is spotted on a chip and probed with a large number of antibodies, is such an approach. They allow researchers to track the activation or perturbation of cellular pathways, to monitor expression of disease-related proteins, and to investigate the cellular effects of drugs, all using very small amounts of sample. Zeptosens in Witterswil, Switzerland, for example, has developed the ZeptoMark CeLyA protein-profiling system, a sensitive reverse array that uses the company's planar waveguide technology. Each chip can contain the protein content of up to 384 different cell lysates for high-throughput profiling.

"We thought that after we had identified how many genes, how many proteins, the problem would go away," says Celis. "But there are protein-protein interactions that give you different functions. Unless you

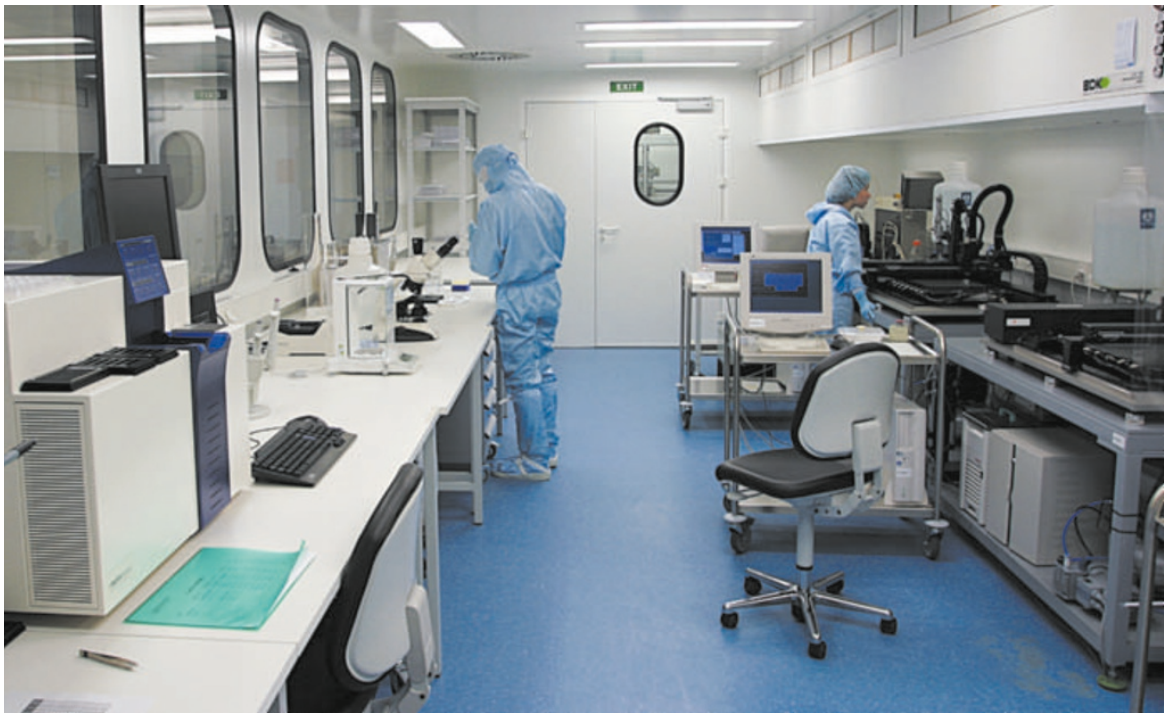

Zeptosens: microarrays must be made under rigorous clean-room conditions.

know those differences, it is difficult to come up with a drug therapy," he points out.

Giulio Superti-Furga, vice-president of biology at drug-development company Cellzome in Heidelberg, Germany, says: "We look at proteins in their own juice, with their own partners, with their own post-translational modifications." Cellzome uses two technologies based on liquid chromatography/tandem mass spectrometry to study the protein context of drugs and drug-like molecules. Potential drug compounds are immobilized and used as affinity reagents to identify binding proteins. Candidate drug targets are then 'positioned' onto cellular pathways using tandem affinity purification, originally developed by researchers at the European Molecular Biology Laboratory in Heidelberg. "You can almost derive an organizational chart, as you'd draw for a company, but for a proteome, with its drug-binding components earmarked," says Superti-Furga. Key to the approach is the compilation and visualization of data by informatics. Such maps should help identify points of intersection between different pathways, such as apoptosis and stroke for the TNF- $\alpha$ pathway and lipid metabolism for the APP pathway.

MDS Proteomics in Toronto, Canada, takes a chemiproteomics approach to iden-

\title{
FAMILY BUSINESS
}

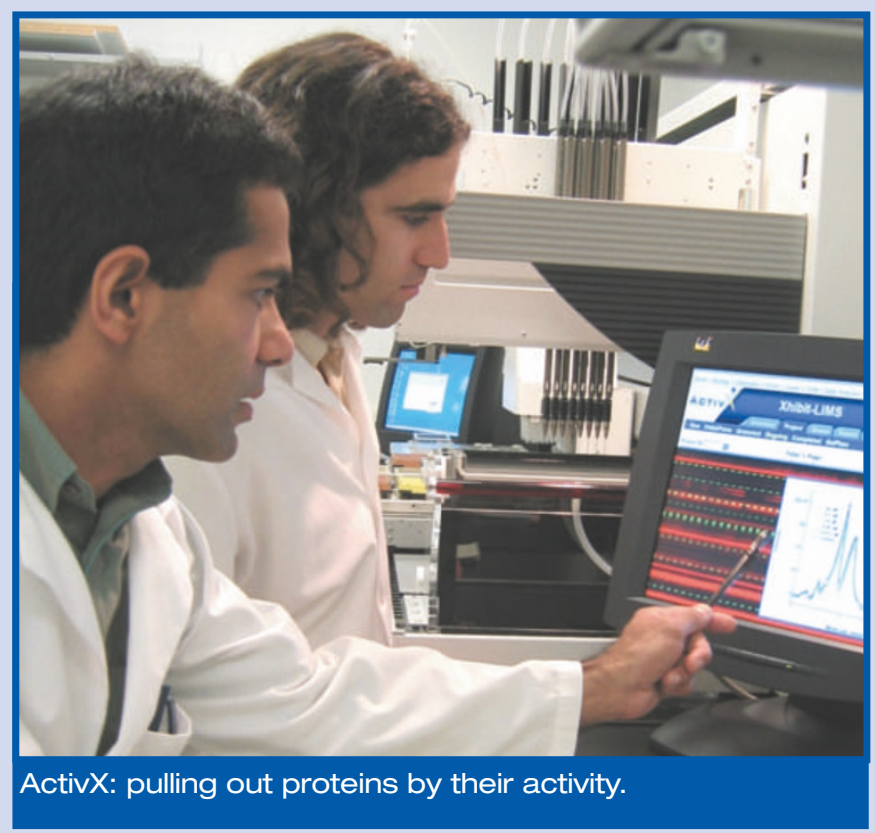

"Suppose you could develop a chemical tool so powerful that you could go into a cell and identify all the members of one family — all the protein kinases, or all the hydrolases or the phosphatases," proposes John Kozarich, president and chief scientific officer at the drug discovery and development company ActivX in La Jolla, California. The activity-based proteomics technology to do just that was originally developed by Ben Cravatt's group at the Scripps Institute and has been commercialized by ActivX.

The approach is based on a simple idea: proteins in the same family tend to have conserved active sites which a well-designed chemical reagent can pick up. In the chemistry literature are thousands of publications probing the structure and functions of proteins, defining inhibitors and ligand interactions. "We harvest that information. We are interested in tools that allow us to look at families related by a common trait," explains Kozarich.

For serine hydrolases, for example, ActivX has designed chemical tools that recognize the underlying catalytic motif - three amino acids in a particular spatial configuration. The system adds a highly fluorescent covalent tag to one of the unique amino-acid residues, and this allows all the serine hydrolases to be pulled out of a sample by capillary electrophoresis, and analysed and identified by mass spectrometry. Early in drug development, a domain scan can compare different animal models to help resolve toxicity issues. Another use is to characterize off-target interactions of drug leads, as the probes are designed to recognize just those protein motifs that are the same as the active sites that are the real drug target. 